\title{
But how thin is a thin wire?
}

\section{Theory and measurement conspire to show that there are quantum limitations of the regularity with} which thin wires conduct electricity.

Aтоміс engineering is not necessarily the craft of designing nuclear reactors. Not so long ago, people were talking optimistically of building microelectronic devices on the scale of interatomic distances, and for obvious reasons: the smaller the devices, the faster will be the arrays into which they are incorporated. But it has always been on the cards that the ambitions of the device constructors would be frustrated by basic difficulties, quantum mechanics for example.

Two neat experiments show that indeed to be the case. It may not be a coincidence that the two groups are from IBM's Yorktown Heights Laboratory (Benoit, A., Umbach, C.P., Laibowitz, R.B. \& Webb, R.A. Phys. Rev. Lett. 58, 2343; 1987) and from AT\&T Bell Laboratories (Skocpol, W.J., Mankiewich, P.M., Howard, R.E., Jackel, L.D. \& Tennant, D.M. Phys. Rev. Lett. 58, 2347; 1987). The Bell team worked with A.D. Stone of Yale University, one of the pioneers in this field.

The question is what happens to an electric current flowing in a wire so thin that quantum effects become important? It is tempting to think of a current as a plane electron wave propagating through the wire, but that cannot be the case when the wire has resistance because electrons are occasionally scattered (losing energy, which is why the wire gets hot). So why not look for quantum interference between scattered electrons? This expectation has been confirmed in the past two years by placing thin wires carrying a constant current in a magnetic field and showing that the measured voltage drop, or measured resistance of the wire, varies with the magnetic field.

But when is a wire properly called "thin"? On the view that a current in a superconducting wire is a plane electron wave travelling along, and that a current in a normal wire is a plane made incoherent by random scattering, a wire can be called thin when its width is less than the length over which the coherence of an electron wave is destroyed by random incoherent scattering. That seems to be the starting point for the measurements now described. They work with conductors with lateral dimensions of a micron or a tenth as much, and even so must use liquid-helium temperatures for the quantum effects to become apparent.

Neither group of authors ducks the interesting question of just what is meant by the measurement of a voltage along the length of such a wire. Obviously, it is not possible to attach voltage probes mechanically to wires made, in one case, by evaporating either gold or antimony by a lithographic process in which a scanning electron microscope was used to etch the pattern. Instead, the probes must be integral with the conductor, which graphically shows that, if the measuring instrument is physically a part of the experiment, measurement can hardly avoid interfering with the phenomenon observed. A voltmeter is a device for measuring the difference of the chemical potential of electrons within the two conducting attachments and must be accessible to electrons and thus contribute to the loss of coherence.

Quite what resistance means is another proper question. Plainly it is not safe to talk about the resistance per unit length of the conductor, but only about the apparent resistance between two specified probes, calculated from Ohm's Law as the ratio of the measured current to the known voltage, thus allowing that the result may be determined by the shapes and other properties of the probes. Both sets of experiments use conductors equipped with at least four built-in probes which can be joined together in six different pairs for voltage measurements. The trick is to span the expected incoherence length with the range of distance separating the possible pairs of probes.

The new result, at which both groups arrive, concerns what happens with the thinnest wires, but the Bell group also neatly confirms what is expected to happen in other circumstances. Given the imponderables, there is no point in simply measuring the resistance between all possible pairs of points; because of quantum interference that will affect the different probes differently, it will not be possible accurately to relate the apparent resistance between a pair of probes with the length of the conductor between them, which is where the random disturbances caused by external magnetic fields are useful: the magnitude of the fluctuation of the voltage between two specified probes is a measure of the extent to which quantum interference affects that part of the system, and is thus a measure of the extent to which a voltage difference is affected by quantum interference even in the absence of a field.

First, there is a simple and almost classical argument. Suppose you have a length of thin wire and place voltage probes supposed not to interfere with the phenomenon observed at equal lengths along it. If there is quantum interference affecting the voltage differences between successive pairs of probes, it will at least be possible to relate the fluctuation along the total length to the fluctuations across the separate pieces by exactly the argument used to show that the standard error of $N$ measurements affected by a source of random error is inversely proportional to the square-root of $N$. Because the voltage will on the average be proportional to the length, the proportional departure of the voltage from expectation will be inversely proportional to the length of the wire. In other words, as common sense suggests, the voltage drop along a long thin wire will be relatively unaffected by the effects of quantum interference at the voltage probes.

The new development is that this appears not to be the case when the distance between the voltage probes is smaller than the incoherence length. Then, remarkably, it seems that the variation of the apparent resistance is constant, and independent of the distance between the probes and thus of the expected value of the resistance. Both groups have shown this by measurement. The IBM group has also inferred that this must be the case by means of a neat use of statistical arguments and an inference about the symmetry of various aspects of the fluctuations with respect to reversals of the magnetic field derived from Onsager's reciprocity relations.

This result is not encouraging for the designers of microelectronic devices. When they embark on making microcircuits whose elements are shorter than the incoherence length, they will discover that mere geometrical length is no longer a good guide to electrical properties. Moreover, it will then emerge that the relative variation of the error due to quantum interference becomes a larger proportion of the total resistance as the length of the circuit element shrinks. It is easy to imagine what disastrous effects this would have on the performance of a microcircuit with, say, a million conducting lengths of thin wire, each with a resistance varying randomly and unavoidably. The only good news is that on the face of things these gloomy speculations should not apply to superconductors.

John Maddox 